\title{
Potential cost to Western Australia of proposed patient co-payments according to healthcare organisational structure: A preliminary analysis
}

\author{
J Alasdair Millar ${ }^{1}$ and Robyn CMillar ${ }^{2}$
}

1. Department of Medicine, Albany Regional Hospital, Albany, WA, Australia \&

Department of Medical Education, Curtin University, Perth, WA, Australia

2. Denmark Health Service, Denmark, WA,Australia

\section{RESEARCH}

Please cite this paper as: Millar JA, Millar RC. Potential cost to Western Australia of proposed patient co-payments according to healthcare organisational structure: A preliminary analysis. AMJ 2014, 7, 8, 357-360. http://doi.org/10.21767/AMJ.2014.2214

Corresponding Author:

Prof J A Millar

Department of Medicine

Albany Regional Hospital

Albany, WA 6330

Australia

Email: Alasdair.millar@health.wa.gov.au

\section{ABSTRACT}

\section{Background}

The Australian federal government has proposed an AUD \$7 patient co-payment for a general practitioner (GP) consultation. One effect of the co-payment may be that patients will seek assistance at public hospital emergency departments (EDs), where currently there is no user charge.

\section{Aim}

We studied the possible financial impact of patient diversion on the Western Australia (WA) health budget.

\section{Method}

We constructed a spreadsheet model of changes in annual cash flows including the co-payment, GP fees for service, and rates of diversion to emergency departments with additional marginal costs for ED attendance.

\section{Results}

Changes in WA cash flows are the aggregate of marginal ED costs of treating diverted patients and added expenditure in fees paid to rural doctors who also man local emergency centres. The estimated costs to WA are AUD $\$ 6.3$ million, $\$ 35.9$ million and $\$ 87.4$ million at 1,5 , and 10 per cent diversion, respectively. Commonwealth receipts increase and expenditure on Medicare benefits declines.

\section{Conclusion}

A diversion of patients from GP surgeries to ED in WA caused by the co-payment will result in increased costs to the state, which may be substantial, and will reduce net costs to the Commonwealth.

\section{Key Words}

Healthcare costs, Patient Medicare co-payment, general practice, public hospitals

\section{What this study adds:}

\section{What is known about this subject?}

The Australian federal government has proposed an AUD \$7 patient co-payment for a GP consultation. The financial impact on states and territories of this proposed AUD \$7 patient co-payment, and the effect on patient flows, are not known.

\section{What new information is offered in this study?}

We have estimated changes to the Commonwealth and WA budgets if the co-payment causes diversion of patients from GP surgeries to public hospital emergency departments.

3. What are the implications for research, policy, or practice?

Our estimates suggest that the proposed co-payment will cause an increase in WA government health expenditure. A detailed model is required to more accurately predict the effect of the co-payment.

\section{Background}

The purpose of the Australian federal government's proposed AUD \$7 co-payment for a GP visit, as announced in the 2014 Budget statement, has been described as generating income, reducing "unnecessary" GP visits, and 
impressing on the public that medical services have a cost. Regardless of the justification, the clinical and fiscal effects of the levy are uncertain. One possible outcome is that patients may be deterred from visiting their GP and will either delay attendance, not attend, or seek medical attention at the local public hospital emergency department, where no charge currently exists in WA. We have studied the financial aspects of the last possibility from the point of view of hospitals in WA and the state and Commonwealth budgets.

\section{Method}

We prepared a spreadsheet model of changes in cash flows between individuals, the state of WA, and the Commonwealth as a function of the proportion of GP patients who divert from GP surgeries to the local hospital $E D$, up to 10 per cent diversion, with separate accounting for the metropolitan area, regional hospitals of the Western Australian Country Health Service (WACHS), and peripheral multipurpose service (MPS) sites, which are smaller rural hospitals attended by local general practitioners on a regulated $^{1,2}$ fee-for-service basis. Health care in remote WA is provided at nursing posts, which we excluded from the analysis. We extrapolated results from our home country region (WACHS Great Southern) to the other six WACHS regions. Our model contains the following assumptions:

1. The fee-for-service schedule used by visiting medical practitioners at MPS sites is complex. We assumed that diverted cases would be of a complexity consistent with a routine GP visit (Level A or B of the WAGMSS system ${ }^{1}$ ) that would therefore not require a visit between midnight and 8am. Patients with acute higher-level illnesses are more likely to already attend emergency departments and thus are less likely to contribute to the marginal costs caused by the diversion. The weighted average of published fees assuming equal distribution between normal and evening working hours and between Levels A and B is AUD \$92.05. ${ }^{2}$ The bulk-billed Medicare fee for a low-level consultation by day is AUD \$36.30.

2. The number of GPs in metropolitan Perth is not reported as a separate line item in public documents and was estimated from the number of clinically active doctors in WA $(5,963)$ and the proportion that are GPs (33.9 per cent) as reported by the Australian Institute of Health and Welfare in 2011, ${ }^{3}$ adjusted by the ratio of the populations of Perth (1.97 million) and WA (2.55 million) (2013 population data) reported by the Australian Bureau of Statistics. ${ }^{4,5}$ The result was also adjusted for leave amongst doctors (10 per cent, equivalent to five weeks leave per year), giving a final figure of 1,395 GPs working on an average day in metropolitan Perth.

3. A marginal utilisation cost per ED attendance of AUD $\$ 50$ was applied. ${ }^{6}$ This figure was reported for NSW in 2002, being 20 per cent of the contemporaneous average ED visit cost. Extrapolation to 2014 dollars would suggest a greater figure, but AUD \$50 was accepted as a reasonable and conservative estimate considering the current average cost per ED attendance at Albany of AUD $\$ 305$ and the above assumption of lower than average acuity.

4. The co-payment will be administered as a charge on patients at the point of attendance, AUD \$2 of which will be retained by each practice. Alternative processes, such as adjustment of patient Medicare reimbursement or the GP bulk-billed reimbursement, were not considered.

5. The model did not include consideration of copayments for pathology tests or radiology, as also proposed by the federal government.

6. The model applies to weekdays only. This is because many general practices close on weekends or open for restricted hours on Saturdays. Thus most patients who seek urgent attention at the weekend are already obliged to attend hospital emergency departments and will not contribute substantially to new marginal costs.

7. Data for WACHS-Great Southern were assumed to apply equally to all WACHS regions.

A sensitivity analysis was performed over uncertainty in the marginal cost of an extra ED attendance. The study did not obtain individual patient data. Ethical approval was not required.

\section{Results}

Table 1 shows the aggregate results from the base-case model. Patients who divert from GP to ED represent a cost benefit to the Commonwealth because Medicare benefits are avoided but a co-payment is received from those who attend the GP. By contrast, there is a net cost to the state of WA, which in the base-case varies from AUD $\$ 6.3$ million to AUD $\$ 87.4$ million between 1 and 10 per cent diversion, respectively.

Diversion of patients affects workloads and cash flows in different ways at each hospital site, based on differences in medical staffing arrangements. At an MPS site, GPs on duty at the ED will submit attendance fee claims to the WA Health Department for the diverted (as well as non- 
diverted) patients, but salaried staff elsewhere will attend diverted patients as part of their normal job descriptions. As the WAGMSS fees are 8 per cent higher than the Medicare schedule fees and some patients will be seen out of hours (attracting a higher fee), the new cost to the state at MPS sites is greater than the substituted Medicare benefit. As a result, rural GP incomes increase by an estimated AUD \$63, AUD \$119, and AUD \$189 per weekday at 1, 5, and 10 per cent diversion, respectively, compared to reductions of AUD \$9, AUD \$43, and AUD \$85 (regional GPs), or AUD \$9, AUD $\$ 51$, and AUD \$132 (metropolitan GPs), respectively. All sites will incur additional marginal costs for emergency department attendance. The model predicts a total diversion across WA of 355, 2,106 and 5,418 patients daily at 1,5 , and 10 per cent diversion rates, respectively.

Table 1: Base-case modelled changes with AUD \$7 payment co-payment

\begin{tabular}{|c|c|c|}
\hline Diversion & Western Australia & Commonwealth \\
\hline $0 \%$ & $\$ 0$ & $\$ 46,327,500$ \\
\hline $1 \%$ & $-\$ 6,305,564$ & $\$ 49,227,602$ \\
\hline $5 \%$ & $-\$ 35,851,449$ & $\$ 63,534,599$ \\
\hline $10 \%$ & $-\$ 87,427,252$ & $\$ 90,585,142$ \\
\hline
\end{tabular}

Base-case modelled changes in Western Australian and Commonwealth cash flows per annum if an AUD \$7 patient co-payment causes a shift of patients from GP surgeries to public hospital emergency departments. The increase in Commonwealth income arises from co-payment receipts (less AUD \$2 assigned to general practices) and, where patients divert to EDs, reduced outlays for Medicare benefits. The negative cash flow in the state arises from increased fees to country GPs and aggregate marginal costs of attendance at public hospital emergency departments (negative cash flow represents added costs).

Table 2 shows the result of sensitivity analysis around emergency department marginal costs.

Table 2: Sensitivity analysis around marginal ED costs

\begin{tabular}{|c|c|c|c|}
\hline \multirow{2}{*}{$\begin{array}{l}\text { New value } \\
\text { for marginal } \\
\text { ED cost }\end{array}$} & \multicolumn{3}{|c|}{ Diversion from GP to ED (\%) } \\
\cline { 2 - 4 } & $1 \%$ & $5 \%$ & $10 \%$ \\
\hline$\$ 25$ & $\$ 3,717,642$ & $\$ 20,472,631$ & $\$ 47,798,600$ \\
\hline$\$ 100$ & $\$ 11,641,602$ & $\$ 65,745,691$ & $\$ 158,908,734$ \\
\hline
\end{tabular}

Sensitivity analysis around marginal ED costs showing the net cost to WA at 1, 5, and 10 per cent diversion, respectively, from GP surgeries to public hospital emergency departments (compare with Table 1). The base-case marginal cost (AUD \$50) was reset to either AUD \$25 or AUD $\$ 100$.

\section{Discussion}

Our results suggest that a significant cost to the WA government will arise if patients seek to avoid the proposed co-payment by attending a public hospital emergency department. This finding is not unexpected, as any increase in demand is likely to be associated with added costs, but the degree of cost disadvantage to the state may be substantial. In addition, we find that:

1. The Commonwealth benefits from diversion from GP services regardless of site because the number of Medicare payments declines, and the co-payment for undiverted patients is credited. The change in Commonwealth receipts shown in Table 1 does not include the proposed laboratory or radiology copayments.

2. Where EDs have salaried staff, surrounding GPs face a reduction of income, but at rural MPS sites, incomes will increase as a result of substitution of WAGMSS payments for Medicare benefits, the former being greater. Though the total number of consultations remains the same, a source of inefficiency will arise from the need for additional travel between the practice and the local hospital.

3. Regional and metropolitan emergency departments will be faced with increased demand, causing further congestion to already overcrowded emergency departments and compromising achievement of targets for ED wait times. Additional hidden costs are likely under this scenario.

We conclude that the likely consequence of the AUD \$7 copayment proposed in the federal budget will be a reciprocal change of costs in WA and the Commonwealth, depending on how hospital services are structured and the extent of patient diversion. Our model has limitations as it takes a simple view of how the co-payment might be administered and contains uncertainties over the actual number of GPs at work in the metropolitan area and the real marginal cost of an ED attendance in WA. It assumes that the AUD \$7 copayment will be collected at the time of each consultation; that is, it sets the number of private consultations at zero and ignores possible exemptions. While the direct remittance of AUD \$5 per case to the Commonwealth is a possible process, the method of securing the co-payment, assuming it passes legislative hurdles, remains uncertain.

We have ignored the proposed levies for pathology and radiology, but these are also likely to amplify any tendency for patients to divert to emergency departments. We have not studied the possibility that the state government will 
respond in kind, and impose a co-payment for attendance at public hospital EDs. Such co-payment would reduce the rate of diversion, but in view of the other two proposed copayments, perhaps not eliminate it. The diversion of patients studied here will be an extension of current realities, as it is widely recognised that patients with nonemergency complaints currently attend EDs across WA and that cost avoidance is likely to be a factor in this behaviour. It is therefore not unreasonable to assume that further diversion to EDs will be a consequence of the co-payment.

\section{Conclusion}

Our base-case estimates contain known (fees for service) and uncertain data. We have performed a sensitivity analysis to mitigate the uncertainty over marginal costs and note in particular that real marginal ED costs will tend to increase at higher rates of diversion. For these reasons we regard our analysis simply as an initial "sighting shot" that suggests likely cost implications of the co-payment for the WA government, but contains residual uncertainty as to their true extent. Further development of our model is justified.

\section{References}

1. Western Australian Government Medical Services Schedule [Internet]. WA Department of Health, 2013. [cited 2014 July 1]. Available from: http://www.health.wa.gov.au/vmp/docs/HDWA2013.pdf

2. Visiting Medical Practitioners: Western Australian Government Medical Services Schedule (WAGMSS) [Internet]. WWA Department of Health, 2014 [revised 2004 Apr 7; cited 2014 Jul 1]. Available from: http://www.health.wa.gov.au/vmp/

3. Australian Institute of Health and Welfare. Medical Workforce 2011. (National Health Workforce Series No.3) [Internet]. [cited 2014 Aug 13]. Available from: http://www.aihw.gov.au/WorkArea/DownloadAsset.as px?id=60129542629

4. Australian Bureau of Statistics. 3101.0-Australian Demographic Statistics, Dec 2013; Latest issue released 19/06/2014 [Internet]. (cited 2014 Aug 13) Available from: http://www.abs.gov.au/ausstats/abs@.nsf/mf/ 3101.0

5. Australian Bureau of Statistics. 3218.0 - Regional Population Growth, Australia, 2012-13; Latest issue released 03/04/2014 [Internet]. (cited 2014 Aug 13). Available from: http://www.abs.gov.au/ausstats/abs@.nsf/Products/32 18.0 201213 Main+Features Western+Australia?Open Document\#PARALINK4
6. Eager K, Sondalini R, Mazevska, D for the NSW Health Department. The NSW Emergency Department Funding Model [Internet]. [cited 2014 Aug 13]. http://www.docstoc.com/docs/31852185/The-NSWEmergency-Department-Funding-Model.

\section{PEER REVIEW}

Not commissioned. Externally peer reviewed.

\section{CONFLICTS OF INTEREST}

Prof Millar is a member of the Australian Medical Association, but has not participated in the Association's submissions to government regarding patient co-payments. 